\title{
Comparison Between Tilt-Table Testing Results Performed During Different Periods of The Day
}

\author{
Tan Chen Wu, Denise Hachul, Mauricio Scanavacca, Eduardo Sosa \\ São Paulo, SP - Brazil
}

Objective - To evaluate the influences of circadian variations on tilt-table testing (TTT) results by comparing the positivity rate of the test performed during the morning with that of the test performed in the afternoon and to evaluate the reproducibility of the results in different periods of the day.

Methods - One hundred twenty-three patients with recurrent unexplained syncope or near-syncope referred for TTT were randomized into 2 groups. In group I, 68 patients, TTT was performed first in the afternoon and then in the morning. In group II, 55 patients, the test was performed first in the morning and then in the afternoon.

Results - The TTT protocol was the prolonged passive test, without drug sensitization. Twenty-nine (23.5\%) patients had a positive result in at least one of the periods. The positivity rate for each period was similar: 20 (16.2\%) patients in the afternoon and $19(15.4 \%)$ in the morning $(p=1.000)$. Total reproducibility (positive/positive and negative/negative) was observed in 49 (89\%) patients in group I and in 55 (81\%) in group II. Reproducibility of the results was obtained in 94 (90.4\%) patients with first negative tests but in $10(34 \%)$ patients with first positive tests.

Conclusion - TTT could be performed during any period of the day, and even in the 2 periods to enhance positivity. Considering the low reproducibility rate of the positive tests, serial TTT to evaluate therapeutic efficacy should be performed during the same period of the day.

Key words: tilt-table testing, circadian variation, vasovagal syncope

Heart Institute of São Paulo University

Mailing address: Denise Hachul - InCor - Unidade Clínica de Arritmia - Av. Dr. Enéas de Carvalho Aguiar, 44 - 05403-000 - São Paulo, SP - Brasil - E-mail: dhachul@incor.usp.br
Vasovagal or neurally mediated syncope is the most common cause of syncope, especially if no evidence is present of underlying structural heart disease ${ }^{1-4}$. Since the first study of Kenny et al ${ }^{5}$, tilt-table testing (TTT) has become a widely used method for diagnosing neurocardiogenic syncope, a type of vasovagal syncope resulting from a "paradoxic" reflex withdrawal of sympathetic tone and vagal activation mediated by orthostatic stress. Although widely used, TTT lacks protocol standardization ${ }^{6-10}$. Due to methodological variations, estimated sensitivity varies from 21 to $74 \%$ and specificity from $70 \%$ to $100 \%$.

Apart from these methodological constraints, circadian variations or chronobiologic factors related to autonomic activity certainly play a role in the clinical manifestation of symptoms and could result in a higher susceptibility to syncope in some periods of the day, and similarly influence positivity and the reproducibility rate of the test ${ }^{11}$.

The aims of the present study were 1) to assess the positivity rate of TTT performed during the afternoon and compare it with to the test performed during the morning and 2) to evaluate the reproducibility of the results when TTT is performed during the 2 periods of the day.

\section{Methods}

The study enrolled 123 patients referred for TTT due to recurrent episodes of unexplained syncope ( $\geq 2$ episodes), between March 1996 and June 1998. All patients had previously undergone clinical examination, electrocardiography, echocardiography, 24-h Holter monitoring, and treadmill exercise testing without elucidation of symptom etiology. No significant structural heart disease had been disclosed. Other invasive procedures, such as electrophysiologic study or coronary angiography, were performed in some patients when required. Referral to a neurologist was accomplished if clinically indicated. The patients were then divided into 2 groups: group I (afternoon/morning), 55 patients, TTT was performed at first in the afternoon (between 1 and 5:00 p.m.) followed by another TTT in the morning (between 8 a.m. and 12 noon). Group II (morning/afternoon), 
68 patients, underwent an initial TTT in the morning and the second one in the afternoon.

Patients were required to fast for at least 6 hours. The examination was performed in a dim, quiet room with little surrounding noise. All medications were discontinued for at least 5 half-lives. No drugs or provocative maneuvers were used to increase test sensitivity.

A tilt table with a footboard support and manual control (Carci Ind. e Com., São Paulo, SP) and a tilting angle of $60^{\circ}$ was used. Blood pressure (BP) was continuously monitored with a noninvasive beat-to-beat determination of finger arterial blood pressure (2300 - Finapress, Ohmeda Medical Systems. Madison, WI, USA). The continuous electrocardiogram as well as blood pressure curves were registered using a polygraph (Hewlett-Packard Co. Andover, Mass., USA) and also recorded automatically with software created specifically for the TTT (Labele System) at the Heart Institute.

After 20 minutes in the supine position, patients were tilted to a $60^{\circ}$ upright position. The endpoints of the test were the occurrence of symptoms (presyncopal or syncopal events) associated with a drop in systolic blood pressure > $30 \mathrm{mmHg}$, a decrease in heart rate, or both of these, or the completion of 40 minutes. According to blood pressure and heart rate changes, positive test responses were classified as the following: 1) vasodepressor response - a marked decrease in BP without a significant heart rate decrease; 2) mixed response - hypotension followed by a decrease in heart rate; 3 ) cardioinhibitory response - sinus pause $>3$ seconds without an antecedent decrease in BP.

The Student $t$ test was used to compare both groups regarding age, time elapsed from the first episode of syncope, frequency of syncope per year, and time interval between the examinations. To compare the number of previous syncopal episodes, a Mann-Whitney test was used. Sex distribution was compared with Pearson's chi-square test.

The Fisher exact test was used to compare the groups regarding general distribution of TTT results in the 2 periods, whether the test was positive in both periods, positive/ positive (PP); positive in only one of the periods, positive/ negative (PN) or negative/positive (NP); or negative in both periods, negative/negative (NN). The McNemar test was used to determine whether the distribution of positive results during the morning and afternoon period were the same.

The variable positivity rate was created to assess whether the patient had at least 1 positive result in the 2 examinations.

The Pearson chi-square test and the Fisher exact test were used to compare the distribution of positive results between male and female sexes and between adults and nonadults (infants/adolescents). Additionally, these tests were used to compare the sex and age distribution of patients with discordant results. The Mann-Whitney test was used to compare the reproducibility of the following variables: number of syncopal episodes, time elapsed from the first episode of syncope, and time interval between the examinations.
Results were considered of statistical significance when a $p$ value $<0.05$ was achieved. The statistical analysis was performed with the usual software (SAS - statistical analysis system) ${ }^{12}$.

\section{Results}

Group I consisted of 55 patients, and group II consisted of 68 patients. Data regarding patients characteristics (age, sex, number of previous syncopal episodes, time elapsed from the first episode of syncope, and time interval between the examinations) are shown in table I. No statistical difference was found between the groups.

When the 2 groups were compared regarding the general distribution of results obtained in both periods, ie, positive/positive (PP), positive/negative (PN), negative/positive (NP), and negative/negative (NN), no significant difference was observed $(\mathrm{p}=0.20)$ (table II).

Among the 123 patients, 29 (23.5\%) had a positive TTT in at least one of the periods, either in the morning or in the afternoon. When the periods of the day were separately analyzed, the number of positive results was similar, with 20 (16.2\%) of the positive tests occurring in the afternoon, and 19 (15.4\%) in the morning (McNemar test, $\mathrm{p}=1.0)$.

The percentage of positive responses in the younger population or $\leq 18$ years $(40.1 \%)$ was higher than that in the adults $(18.7 \%)(\mathrm{p}=0.02)$. No significant difference existed in the rate of positive response between males and females $(\mathrm{p}=0.56)$.

A total of 104 (84.5\%) patients had the same results in both tests. Total reproducibility between the 2 groups was not statistically different. In group 2, results were concordant in 55 (81\%) patients and in group I, in $49(89 \%)$ patients (table II).

Positive responses were reproduced in just 10 (34.4\%) of 29 patients and negative responses were reproduced in 94 (90.4\%) of 104 patients (table II). The reproducibility of TTT when the first test was negative, regardless of the period of the day, was estimated in $94 \%$ (94 out of 100 patients), whereas the probability of obtaining an identical result when the first test was positive was $43.5 \%$ ( $10 \mathrm{of} 23 \mathrm{pts}$ ) (table III).

\begin{tabular}{|c|c|c|c|}
\hline \multicolumn{4}{|c|}{$\begin{array}{l}\text { Table I - Comparison among data regarding sex, age, mean number } \\
\text { of syncopal episodes, time elapsed from the first syncopal episode and } \\
\text { time interval between the examination for groups I and II }\end{array}$} \\
\hline \multirow[b]{2}{*}{ Variable } & \multicolumn{2}{|c|}{ Group } & \multirow[b]{2}{*}{$\mathrm{p}$ value } \\
\hline & I & II & \\
\hline Sex (female) & $38(69 \%)$ & $52(76.4 \%)$ & $\mathrm{p}=0.36$ \\
\hline Minimum & 5 years & 5 years & \\
\hline Age maximum & 68 years & 86 years & \\
\hline (mean) & $(30.2 \pm 14)$ & $(35.1 \pm 20)$ & $\mathrm{p}=0.12$ \\
\hline Syncopal episodes (mean) & $4.5 \pm 2.8$ & $5.4 \pm 4.3$ & $\mathrm{p}=0.61$ \\
\hline $\begin{array}{l}\text { Time elapsed from the first } \\
\text { episode (years) }\end{array}$ & $8.3 \pm 8.6$ & $6.3 \pm 8.7$ & $\mathrm{p}=0.21$ \\
\hline $\begin{array}{l}\text { Frequency of syncope in a year } \\
\quad \text { (mean/median) }\end{array}$ & $2.7 / 1.1$ & $4.6 / 1.0$ & $\mathrm{p}=0.28$ \\
\hline $\begin{array}{r}\text { Time interval between the } \\
\text { exams (mean in days) }\end{array}$ & $33 \pm 2.8$ & $9.4 \pm 16.4$ & $\mathrm{p}<0.001$ \\
\hline
\end{tabular}




\begin{tabular}{|c|c|c|c|c|c|c|c|}
\hline \multicolumn{8}{|c|}{$\begin{array}{c}\text { Table II - Distribution of TTT results in the morning and } \\
\text { afternoon }\end{array}$} \\
\hline \multirow[b]{2}{*}{ Results } & \multicolumn{5}{|c|}{ Group } & \multirow{2}{*}{\multicolumn{2}{|c|}{ Total }} \\
\hline & & & I & & & & \\
\hline Morning & Afternoon & $\mathrm{N}$ & $\%$ & $\mathrm{~N}$ & $\%$ & $\mathrm{~N}$ & $\%$ \\
\hline $\mathrm{N}$ & $\mathrm{N}$ & 44 & 80 & 50 & 74 & 94 & 76.4 \\
\hline$P$ & $\mathrm{P}$ & 5 & 9.1 & 5 & 7.4 & 10 & 8.1 \\
\hline$P$ & $\mathrm{~N}$ & 1 & 1.8 & 8 & 11.8 & 9 & 7.3 \\
\hline $\mathrm{N}$ & $\mathrm{P}$ & 5 & 9.1 & 5 & 7.4 & 10 & 8.1 \\
\hline Total & & 55 & 100 & 68 & 100 & 123 & 100 \\
\hline
\end{tabular}

\begin{tabular}{|c|c|c|c|c|c|c|c|}
\hline \multicolumn{8}{|c|}{$\begin{array}{l}\text { Table III - Distribution of TTT results according to their sequence } \\
\text { of presentation (first or second) }\end{array}$} \\
\hline \multirow{2}{*}{\multicolumn{2}{|c|}{ Sequence }} & \multicolumn{4}{|c|}{ Group } & \multirow{2}{*}{\multicolumn{2}{|c|}{ Total }} \\
\hline & & \multicolumn{2}{|c|}{ I } & \multicolumn{2}{|c|}{ II } & & \\
\hline $1^{\circ}$ & $2^{\circ}$ & $\mathrm{N}$ & $\%$ & $\mathrm{~N}$ & $\%$ & $\mathrm{~N}$ & $\%$ \\
\hline $\mathrm{N}$ & $\mathrm{N}$ & 44 & 80 & 50 & 74 & 94 & 76.4 \\
\hline$P$ & $\mathrm{P}$ & 5 & 9.1 & 5 & 7.4 & 10 & 8.1 \\
\hline$P$ & $\mathrm{~N}$ & 5 & 9.1 & 8 & 11.8 & 13 & 10.6 \\
\hline $\mathrm{N}$ & $P$ & 1 & 1.8 & 5 & 7.4 & 6 & 4.9 \\
\hline Total & & 55 & 100 & 68 & 100 & 123 & 100 \\
\hline
\end{tabular}

No statistical difference was observed in the reproducibility of the tests among the variables analyzed, $86.7 \%$ for females and $78 \%$ for males $(\mathrm{p}=0.28)$ and $87.5 \%$ for adults and $74.1 \%$ for children/adolescents ( $\mathrm{p}=0.13$ ). Additionally, no significant differences were observed regarding the number of prior syncopal episodes $(\mathrm{p}=0.59)$, time elapsed from the first syncopal episode $(\mathrm{p}=0.41)$, and time interval between the 2 tests $(p=0.22)$.

The types of hemodynamic responses in a positive TTT for both groups are described in table IV 4. Most responses weremixed or vasodepressor. The type of hemodynamic response was reproduced in all 10 patients with positive results. The mean time to achieve a positive response in the morning was $17.68 \pm 10.31$ minutes and $24.35 \pm 7.58$ minutes in the afternoon. In the 10 patients with reproducible positive results, the mean time for a positive response was $15.30 \pm 9.43$ minutes in the morning and $24.60 \pm 8.22$ minutes in the afternoon.

Regarding the sequence of the test, the mean time for a positive response to occur in the first TTT was $18.80 \pm$ 10.83 minutes, and $21.11 \pm 9.18$ minutes for the second test. It should be noted that a trend toward a delayed positive response occurred when the test was performed in the afternoon period, regardless of the sequence of the test (table V). Nevertheless, this estimation is statistically irrelevant as each subgroup had only a small number of patients.

\section{Discussion}

In this study, no differences were observed regarding the reproducibility of the positivity rate of TTT when

\begin{tabular}{|c|c|c|c|c|}
\hline \multicolumn{5}{|c|}{$\begin{array}{l}\text { Table IV - Comparison of positive results regarding the time for a } \\
\text { positive response and period of day to occur }\end{array}$} \\
\hline Group & Morning & Time (min) & Afternoon & Time (min) \\
\hline \multirow[t]{18}{*}{ I } & PV & 25 & PV & 30 \\
\hline & $\mathrm{N}$ & & $\mathrm{PM}$ & 31 \\
\hline & $\mathrm{N}$ & & PV & 21 \\
\hline & PV & 30 & $\mathrm{~N}$ & \\
\hline & PV & 15 & PV & 10 \\
\hline & PV & 21 & $\mathrm{PV}$ & 28 \\
\hline & $\mathrm{N}$ & & PV & 11 \\
\hline & $\mathrm{N}$ & & PV & 23 \\
\hline & PM & 5 & PM & 27 \\
\hline & $\mathrm{PM}$ & 18 & $\mathrm{PM}$ & 24 \\
\hline & $\mathrm{N}$ & & $\mathrm{PC}$ & 24 \\
\hline & PV & 20 & PV & 26 \\
\hline & PM & 2 & $\mathrm{PM}$ & 35 \\
\hline & PM & 5 & $\mathrm{~N}$ & \\
\hline & $\mathrm{PM}$ & 33 & $\mathrm{~N}$ & \\
\hline & PM & 30 & $\mathrm{~N}$ & \\
\hline & $\mathrm{N}$ & & PM & 34 \\
\hline & $\mathrm{N}$ & & PV & 21 \\
\hline \multirow[t]{11}{*}{ II } & $\mathrm{PV}$ & 14 & $\mathrm{~N}$ & \\
\hline & $\mathrm{PM}$ & 2 & $\mathrm{~N}$ & \\
\hline & $\mathrm{PM}$ & 24 & $\mathrm{~N}$ & \\
\hline & $\mathrm{N}$ & & PM & 35 \\
\hline & PV & 31 & PV & 35 \\
\hline & PV & 6 & $\mathrm{PV}$ & 15 \\
\hline & PV & 10 & PV & 17 \\
\hline & $\mathrm{N}$ & & PV & 21 \\
\hline & PM & 25 & $\mathrm{~N}$ & \\
\hline & PM & 20 & $\mathrm{~N}$ & \\
\hline & $\mathrm{N}$ & & PM & 20 \\
\hline
\end{tabular}

\begin{tabular}{|l|ll|}
\hline Table $\mathbf{V}$ - Mean time for a positive response regarding the period \\
of the day and the sequence of the tests
\end{tabular}

performed in the morning and in the afternoon. However, significant differences were observed concerning the reproducibility of positive tests in different periods, possibly due to individual circadian variations in the autonomic tone.

The lack of a "gold standard" to diagnose neurocardiogenic syncope results in difficulties in establishing the sensitivity of TTT ${ }^{13-18}$. In a review of 12 published reports of studies involving 333 patients that underwent passive TTT, Pavri et al ${ }^{19}$ reported that the positivity rate varies from $7 \%$ to $55 \%$ (mean of $24 \%$ ). Linzer et al ${ }^{20}$, after reviewing 425 patients who underwent passive tilting studies, observed that a mean of $45 \%$ had a positive result (range, $26 \%$ to $90 \%$ ) ${ }^{20}$. In a prior study undertaken at our institution, regarding patients with unexplained syncope, we observed positive results in $41 \%$. 
In this study, we observed a low positivity rate compared with our previous observation and the observations made by other authors. It may be explained by the characteristics of the patients selected. We determined as criteria of inclusion every patient referred for to TTT with a history of 2 or more syncope episodes without structural heart disease. However, in both groups we observed a low frequency of syncope per year, and this fact could be attributed as the cause of the low positivity rate observed.

Also in this study, the prevalence of positive results among distinct age groups shows a lower prevalence of neurally mediated syncope in older patients. We have previously shown the prevalence of positive TTT in patients with unexplained syncope to be $40 \%$ for children and $18 \%$ for elder patients ${ }^{22-23}$.

Autonomic nervous system (ANS) hyperactivity is the main cause of neurally mediated syncope ${ }^{24-26}$. A circadian variation of autonomic activity is well known to occur in a healthy population, with predominant sympathetic activity during the day, mainly during the morning, and parasympathetic activity predominantly occurring during the night ${ }^{27-33}$. During sleep, sympathetic activity is reduced, resulting in a decrease in blood pressure and heart rate. On awakening, however, the sympathetic activity increases substantially and is associated with an increase in circulating catecholamine levels, vasomotor tone, and, consequently, increased blood pressure and heart rate. Some cardiovascular events depend at least partly on the ANS and have a clear circadian rhythm, such as sudden death, ectopic ventricular activity, acute myocardial infarction, stroke, episodes of angina pectoris, bursts of hypertension, and the ability of platelets to aggregate ${ }^{34-35}$.

In addition, postural changes, physical activity, and food ingestion also influence ANS variations and certainly influence TTT results and also influence its reproducibility when it is performed in distinct periods of the day. The variation in these responses on different days is not unexpected, because the clinical presentation of vasovagal syncope is also random and unpredictable in nature. Serial TTT has been advocated as a method of assessing therapeutic efficacy in patients with recurrent syncope and a positive test. However, it remains controversial. The reproducibility of a passive TTT with distinct protocols ranges from $62 \%$ to $80 \%$ when these examinations are performed in a 3- to 7-day interval. The reproducibility of a positive TTT is significantly lower (31\% to $71 \%$ ) than a negative TTT $(83 \%$ to $94 \%)$. Brooks et al ${ }^{36}$ have demonstrated an initial positive TTT to have a reproducibility of $31 \%$, whereas Pavri et al ${ }^{19}$ have reported $49 \%$ on 2 consecutive days. Raviele et al ${ }^{37}$ showed reproducibility to be $71 \%$ when the second examination is performed in an average of three 3 days after the first test. Blanc et al ${ }^{38}$, on the other hand, reported reproducibility to be $62 \%$ when the subsequent examination was undertaken after 7 days, but Ruiz et al ${ }^{39}$ demonstrated that a positive TTT response could be reproduced in only $54.5 \%$ of the patients after a mean interval of 9.7 days (range 1 to 35 days). When the examinations were performed at average 7-day intervals, Hachul et al ${ }^{40}$ observed the reproducibility of a positive response to be $82 \%$, reduced to $77.8 \%$ when the same type of hemodynamic response was concerned.

All reports related above were performed mainly during the same period of the day (morning). They are similar to our results regarding total reproducibility $(84.5 \%)$ and reproducibility of negative results $(90.4 \%)$. However, we observe that reproducibility of a positive TTT result in the present study is rather low (34.4\%), even when compared with that of our own previously published data. A variation in vagal tone with a diurnal variation during the day may account for a different test response in the same patient, in a different period during the day.

Analysis of heart rate variability is a widely accepted method used to evaluate the ANS. Several reports have used it to investigate patients with neurally mediated syncope ${ }^{41-43}$. To assess the reproducibility of heart rate variability indexes, Kochiadakis et al ${ }^{44}$ evaluated 35 patients with neurocardiogenic syncope who underwent serial TTT in the morning period, in a 7-day interval. He showed that patients with vasovagal syncope exhibit a significant day-today variation in vagal tone compared with a control group, and that they were more prone to a TTT-induced syncope when an increased parasympathetic activity was present ${ }^{44}$. These findings confirm the presence of periodic individual autonomic tone variations, which in turn may account for the reported TTT reproducibility findings. These data, combined with the well-known ANS variations, might explain the low reproducibility of TTT observed in our patients.

An $8 \%$ increase in positive TTT results could be demonstrated when patients underwent repeat TTT. When repeating the baseline test, this strategy would increase the overall TTT from isolated rates of $16.2 \%$ in the morning and $15.4 \%$ during the afternoon period to $23.5 \%$ for both periods. This observation might be enough to justify performing another test in a distinct period of the day in patients with an initial negative TTT and symptoms strongly suggestive of a neurocardiogenic etiology for syncope to increase diagnostic probability.

Summarizing, no differences could be observed in the general prevalence of positive TTT responses when it was performed in different periods of the day. General reproducibility of results in both periods was comparable to that of previous reports of tests performed during the same period of the day. However, concordance between 2 positive tests in different periods of the day was low. 


\section{References}

1. Kapoor WN. Evaluation and management of patient with syncope. JAMA 1992 268: $2553-60$.

2. Shen WK, Gersh BJ. Syncope: mechanisms, approach, and management. In: Low PA, ed. Autonomic Disorders Evaluation and Management. Boston: Little Brown and Co., 1992: 605-33.

3. Kapoor WN. Diagnostic evaluation of syncope. Am J Med 1991; 90: 91-106.

4. BendittDG, Remole S, Milstein S, Bailin S. Syncope: causes, clinical evaluation, and current therapy. Annu Rev Med 1992; 43: 283-300.

5. Kenny RA, Ingram A, Bayliss J, Sutton R. Head-up tilt: A useful test for investigating unexplained syncope. Lancet 1986; 11: 1352-4.

6. Khurana RK, Nicholas EM. Head-up tilt table test: how far and how long? Clin Auton Res 1996; 6: 335-41.

7. Janosik DL, Genovely H, Fredman C, Bjerregaard P. Discrepancy between headup tilt test results utilizing different protocols in the same patient. Am Heart J 1992; 123: 538-41.

8. Benditt DG, Remole S, Bailin S, Dunnigan A, Asso A, Milstein S. Tilt table testing for evaluation of neurally-mediated (cardioneurogenic) syncope: rationale and proposed protocols. PACE 1991; 14: 1528-37.

9. Rubin AM, Rials SJ, Marinchak RA, Kowey PR. The head-up tilt table test and cardiovascular neurogenic syncope. Am Heart J 1993; 125: 476-82.

10. Sheldon R, Killam S. Methodology of isoproterenol-tilt table testing in patients with syncope. J Am Coll Cardiol 1992; 19: 773-9.

11. Benditt DG, Ferguson DW, Grubb BP, et al. Tilt table testing for assessing syncope. J Am Coll Cardiol 1996; 28: 263-75

12. Rosner B. Fundamentals of Bioestatistics. Michael Payne ed, PWS Publishers, Boston, Massachusetts, USA. 1986: 584p.

13. Henderson MC, Prabhu SD. Syncope: current diagnosis and treatment. Curr Probl Cardiol 1997; 22: 239-96.

14. Fitzpatrick AP, Theodorakis G, Vardas P, Sutton R. Methodology of head-up tilt testing in patients with unexplained syncope. J Am Coll Cardiol 1991; 17: 125-30.

15. Oribe E, Caro S, Perera R, Winters SL, Gomes JA, Kaufmann H. Syncope: The diagnostic value of head-up tilt testing. PACE 1997; 20(Pt. I): 874-9.

16. Grubb BP, Wolfe D, Samoil D, et al. Recurrent Unexplained Syncope in the elderly: The use of Head-upright tilt table testing in evaluation and management. J Am Geriatr Soc 1992; 40: 1123-8.

17. Van de Walle JP, Panagides D, Messier M, et al. Tilt table testing of young adult patients: Improved speed and sensitivity using an isoproterenol bolus and a continuous $60^{\circ}$ tilt. PACE 1998; 21: 494-8.

18. Pongiglione G, Fish FA, Strasburger JF, Benson W. Heart rate and blood pressure response to upright tilt in young patients with unexplained syncope. J Am Coll Cardiol 1990; 16: 165-70.

19. Pavri BB, Ruskin JN, Brooks R. The yield of head-up tilt testing is not significantly increased by repeating the baseline test. Clin Cardiol 1996; 19: 494-6.

20. Linzer M, Yang EH, Mark Estes III NA, Wang P, Vorperian VR, Kapoor WN. Diagnosing syncope part 2: Unexplained syncope. Ann Intern Med 1997; 127: 76-86.

21. Hachul D, Sosa EA, Consolim F, et al. Valor diagnóstico do teste de inclinação na avaliação da síncope de origem indeterminada. Resultados preliminares. Arq Bras Cardiol 1994; 62: 7-9.

22. Baia A, Hachul D, Contin F, Scanavacca M, Sosa E. Valor diagnóstico do teste de inclinação na avaliação de síncope inexplicado em idosos. Reblampa 1998; 11: 148.

23. Wu TC, Hachul D, Scanavacca M, Sosa E. Response to tilt table test in children with recurrent unexplained syncope. J Am Coll Cardiol 1998; 31: 353C.
24. Lurie KG, Benditt D. Syncope and the autonomic nervous system. J Cardiovasc Electrophysiol 1996; 7: 760-76.

25. Morillo CA, Ellenbogen KA, Pava LF. Pathophysiologic basis for vasodepressor syncope. Cardiol Clin 1997; 15: 233-49.

26. Grubb BP, Kosinski D. Disautonomic and reflex syncope syndromes. Cardiol Clin 1997; 15: 257-68.

27. Millar-Craig MW, Bishop CN, Raftery EB. Circadian variation of blood pressure. Lancet 1978; 1: 795-7.

28. Furlan R, Guzzetti S, Crivellaro W, et al. Continuous 24-hour assessment of the neural regulation of systemic arterial pressure and $\mathrm{RR}$ variabilities in ambulant subjects. Circulation 1990; 81: 537-47.

29. Huikuri HV, Kessler KM, Terracall E, Castellanos A, Linnaluoto MK, Myerburg RJ. Reproducubility and circadian rhythm of heart rate variability in healthy subjects. Am J Cardiol 1990; 65: 391-3.

30. Kawano Y, Tochikubo O, Minamisawa K, Miyajima E, Ishii M. Circadian variation of haemodynamics in patient with essential hypertension: comparison between early morning and evening. J Hypertens 1994; 12: 1405-12.

31. Panza JA, Epstein SE, Quyyumi AA. Circadian variation in vascular tone and its relation to a-sympathetic vasoconstrictor activity. N Engl J Med 1991; 325: 1038-9.

32. Huikuri HH, Niemelä MJ, Ojala S, Rantala A, Ikäheimo MJ, Airaksinen J Circadian rhythms of frequency domain measures of heart rate variability in healthy subjects and patients with coronary artery disease. Circulation 1994; 90: 121-6.

33. Freitas J, Lago P, Puig J, Carvalho MJ, Costa O, Freitas AF. Circadian heart rate variability rhythm in shift workers. J Electrocardiol 1997; 30: 39-44

34. Deedwania PC. Hemodynamic changes as triggers of cardiovascular events. Cardiol Clin 1996; 14: 229-38.

35. Quyyumi AA. Circadian rhythms in cardiovascular disease. Am Heart J 1990; 120: 726-33.

36. Brooks R, Ruskin JN, Powell AC, Newell J, Garan H, McGovern BA. Prospective evaluation of day-to-day reproducibility of upright tilt-table testing in unexplained syncope. Am J Cardiol 1993; 71: 1289-92.

37. Raviele A, Gasparini G, Di Pede F, Delise P, Bonso A, Piccolo E. Usefulness of head-up tilt test in evaluating patients with syncope of unknown origin and negative electrophysiologic study. Am J Cardiol 1990; 65: 1322-7.

38. Blanc JJ, Mansourati J, Maheu B, Boughaleb D, Genet L. Reproducibility of a positive passive upright tilt test at a seven-day interval in patients with syncope. Am J Cardiol 1993; 72: 469-71.

39. Ruiz GA, Scaglione J, Zuekgaray JG, Lopes C. Reproducibility of head-up tilt test in patients with syncope. Clin Cardiol 1996; 19: 215-20.

40. Hachul D, Sosa EA, Consolim F, et al. Reprodutibilidade do teste de inclinação em pacientes com síncope neurocardiogênica. Arq Bras Cardiol 1994; 62: 297-9.

41. Lippman N, Stein KM, Lerman BB. Failure to decrease parasympathetic tone during upright tilt predicts a positive tilt-table test. Am J Cardiol 1995; 75: 591-5.

42. Kochiadakis GE, Orfanakis A, Chryssostomakis SI, Manios EG, Kounali DK, Vardas PE. Autonomic nervous system activity during tilt testing in syncopal patients, estimated by power spectral analysis of heart rate variability. PACE 1997; 20(pt.1): 1332-41.

43. Theodorakis GN, Kremastinos DT, Avrambos GT, Stefanakis GS, Karavolias GK, Toutouzas PK. Heart rate variability in patients with vasovagal syndrome. PACE 1992; 15 (Pt 2): 2221-5.

44. Kochiadakis GE, Kanoupakis EM, Rombola AT, Igoumenidis NE, Chlouverakis GI, Vardas PE. Reproducibility of tilt table testing in patients with vasovagal syncope and it relation to variations in autonomic nervous system activity. PACE 1998; 21: 1069-76. 